\title{
Exertional Dyspnoea in a Young Patient Discharged for COVID-19 Related Acute Respiratory Failure: What is Going on?
}

\author{
Poggiali Erika*, Antonini Mariachiara, Buttafava Federico, Ferrari Valentina, Vercelli Andrea and \\ Magnacavallo Andrea
}

Emergency Department, Guglielmo da Saliceto Hospital, Piacenza, Italy

"Corresponding author: Erika P, MD, Emergency Department, Guglielmo da Saliceto Hospital, Piacenza, via Taverna 49, 29121, Italy, Tel: +39 05320 303044; E-mail: poggiali.erika@ gmail.com / E.Poggiali@ausl.pc.it

Received: May 04, 2020; Accepted: May 12, 2020; Published: May 20, 2020

\begin{abstract}
Coronavirus disease 2019 (COVID-19) can cause severe interstitial pneumonia and acute respiratory failure with the need of non-invasive ventilation or intubation. Particular attention should be done to patients discharged with the diagnosis of COVID-19 acute respiratory failure. Medical and instrumental follow-up should be planned at discharge because of possible chronic sequelae due to persistent pulmonary damage, including pulmonary fibrosis and the development of pulmonary hypertension and right-sided heart failure (cor pulmonale), particularly in young patients. In addition, prothrombotic state must be always considered in these patients both during hospitalization and after being released. We describe the case of a 54-year-old man who was admitted to our Emergency Department with persistent dyspnoea on exertion after being discharged for severe COVID-19 pneumonia from another COVID-19 hospital with a single negative nasopharyngeal swab for 2019-nCOV.
\end{abstract}

Keywords: COVID-19; Nasal-pharyngeal swab; Pneumonia; Acute respiratory failure; Pulmonary embolism; Exertional dyspnoea; Interstitial disease

\section{Learning Point}

- Coronavirus disease (COVID-19) can cause interstitial pneumonia and acute respiratory failure

- Pulmonary embolism should be investigated in COVID-19 patients with dyspnoea on exertion even in presence of a negative nasopharyngeal swab

- Cardiological and pulmonary follow-up should be planned over time for the diagnosis and the management of possible COVID-19 related pulmonary fibrosis, pulmonary hypertension and right-sided heart failure

Citation: Erika P, Mariachiara A, Federico B, et al. Exertional Dyspnoea in a Young Patient Discharged for COVID-19 Related Acute Respiratory Failure: What is Going on? Clin Case Rep Open Access. 2020;3(2):146. 
www.yumedtext.com | May-2020 | ISSN: 2582-5038 | https://dx.doi.org/10.46527/2582-5038.146

\section{Introduction}

Coronavirus disease 2019 (COVID-19) can have several clinical scenarios: from flu-like symptoms with fever, cough and sore throat to gastrointestinal issues until acute respiratory distress syndrome (ARDS) and multi-organ failure [1,2]. Risks factor for ARDS are older age, cardiovascular diseases and diabetes, but also laboratory abnormalities, such as lymphopenia, elevated inflammatory indices (C-reactive protein, serum ferritin), and organ (aspartate aminotransferase, albumin, creatinine, urea, lactate dehydrogenase) and coagulation dysfunction (prothrombin time and D-dimer) [3]. Diagnosis of COVID-19 is based on Real-Time PCR using nasopharyngeal swab and imaging findings, including lung ultrasound (LUS) and chest computed tomography (CT) scan [4,5]. CT findings are important for both diagnosis and follow-up [6]. COVID-19 may predispose patients to thrombotic disease due to excessive inflammation, platelet activation and endothelial dysfunction [7,8]. At time of writing, there are no data on cardiological and pneumological follow-up of patients hospitalized for COVID-19 pneumonia or ARDS.

\section{Case Report}

A 54-year-old man was admitted to our Emergency Department (ED) complaining dyspnoea on exertion over the previous 2 weeks, since he was released from another COVID-19 hospital.

He was hospitalized at the end of March 2020 in our hospital for fever (body temperature at admission $38.5^{\circ} \mathrm{C}$ ) and acute respiratory failure with a need for high-flow oxygen therapy ( $\mathrm{SpO} 291 \%$ at ambient room, $\mathrm{PaO} 2 / \mathrm{FiO} 2100 \mathrm{mmHg}$ ). $\mathrm{His}$ past medical history was unremarkable. He was a no smoker with an active life, and he did not take any medication. He was a bartender, married with a healthy woman with no respiratory symptoms nor fever. At admission point-of-care LUS documented diffuse bilateral B lines in absence of pleural effusion. High resolution chest CT scan showed diffuse ground glass opacities with initial crazy-paving pattern and visual score of $60 \%$ (FIG. 1). A nasopharyngeal swab confirmed the diagnosis of COVID-19 pneumonia. In presence of high respiratory rate (50 breaths/minute) and acute respiratory failure, Non-Invasive Ventilation with Positive End Expiratory Pressure (CPAP NIV) with Stardmed Helmet was immediately started in the Emergency Room with good patient's compliance. He was hospitalized and treated with darunavir/cobicistat, hydroxychloroquine, systemic corticosteroids, Low Molecular Weight Heparin (enoxaparin) at anticoagulant dose and empiric antibiotics (azithromycin and ceftriaxone). Tuberculosis, HIV, hepatitis C and B were excluded by blood tests. Serial blood cultures resulted always negative. Repeated serum procalcitonin remained always in the normal range. After 5-day therapy, fever completely solved. The patient was treated with NIV/CPAP, alternating with High Flux Reservoir Mask for 13 days, with progressive improvement of gas exchange (reduced oxygen requirement and reduced ventilation demand). He was transferred to another COVID-19 hospital for the need of beds in sub-intensive and intensive care units due to the overcrowding of our ED for the massive flux of critically ill COVID-19 patients. His medical condition progressively improved. LMWH therapy was shifted to prophylactic dose in the last 4 days of recovery, then stopped at discharge. A single nasopharyngeal swab collected the day before his discharge was negative, so he was considered healed and released after 23 days of hospitalization in good health, but with persistent dyspnoea on exertion. Chest CT scan was not repeated. Laboratory findings at admission and discharge are reported in TABLE 1. No medical treatment was prescribed, nor medical follow-up was planned at discharge. 


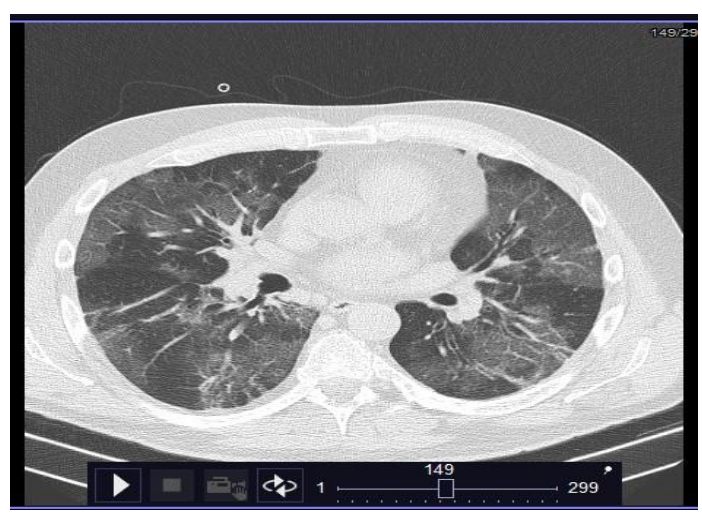

FIG. 1. Lung CT scan. The image shows multiple ground-glass opacities with initial crazy paving pattern with visual score of $60 \%$.

TABLE 1. Patient's laboratory findings at admission, discharge and re-admission.

\begin{tabular}{|c|c|c|c|}
\hline & At admission & At discharge & At re-admission \\
\hline WBC $\left(4-10 \times 10^{9} / L\right)$ & 8.56 & 6.49 & 4.95 \\
\hline Neutrophil Count $\left(2-8 \times 10^{9} / \mathrm{L}\right)$ & 7.61 & 4.35 & 2.50 \\
\hline Lymphocyte Count $\left(1.5-4 \times 10^{9} / \mathrm{L}\right)$ & 0.68 & 1.13 & 1.93 \\
\hline PLT Count $\left(150-450 \times 10^{9} / \mathrm{L}\right)$ & 163 & 237 & 223 \\
\hline PT-INR & 1.27 & ND & 1.09 \\
\hline PTT sec & 34.6 & ND & 30.1 \\
\hline CRP $(<0.5 \mathrm{mg} / \mathrm{dL})$ & 30.97 & 0.82 & 0.74 \\
\hline PCT $(<0.5 \mathrm{ng} / \mathrm{mL})$ & 0.17 & 0.15 & ND \\
\hline Fibrinogen (150-400 mg/dL) & ND & ND & 418 \\
\hline D-dimer $(<500$ ng/mL $)$ & 5699 & ND & 5047 \\
\hline Troponin I (<31 ng/L) & ND & ND & 3.9 \\
\hline LDH (0-248 U/L) & 640 & ND & 261 \\
\hline CPK (0-172 U/L) & 514 & 60 & 33 \\
\hline Serum ferritin $(150-400 \mathrm{ng} / \mathrm{mL})$ & 1301 & ND & ND \\
\hline IL-6 $(<6.4 \mathrm{pg} / \mathrm{mL})$ & ND & 4.1 & ND \\
\hline Creatinine (0.6-1.2 mg/dL) & 0.96 & 0.97 & 0.86 \\
\hline $\begin{array}{c}\text { Blood urea nitrogen } \\
(10-50 \mathrm{mg} / \mathrm{dL})\end{array}$ & 29 & 47 & 35 \\
\hline AST (10-37 U/L) & 132 & ND & 18 \\
\hline ALT (10-37 U/L) & 112 & ND & 27 \\
\hline GGT (7-40 U/L) & 334 & ND & ND \\
\hline Total bilirubin (0.1-1.1 mg/dL) & 1.0 & 0.3 & 0.54 \\
\hline $\mathrm{PaO2} / \mathrm{FiO2}$ (mmHg) & 100 (room air) & ND & 326 \\
\hline SIC score $(>=4)$ & 3 & ND & 0 \\
\hline RT-PCR NP swab & positive & negative & positive \\
\hline
\end{tabular}


WBC, white blood cell count; PLT, platelet; PT, prothrombin time; PTT, partial thromboplastin time; CRP, C-reactive protein; PCT, procalcitonin; LDH, Lactate dehydrogenase; CPK, creatine phosphokinase; IL-6, interleukin-6; AST, aspartate transaminase; ALT, alanine transaminase; GGT, Gamma-glutamyltransferase; SIC, sepsis-induced coagulopathy (https://www.mdcalc.com/covid-19). NP, nasopharyngeal. ND, not done. Abnormal values are in red.

When we evaluated the patient, he was in good clinical condition. Physical examination did not reveal abnormalities. Blood pressure, body temperature, respiratory and cardiac rate were all normal. He denied fever, cough and chest pain, but he complained of exertional dyspnoea, that rapidly solved at rest, since his release from the hospital. Blood arterial gas at room ambient showed normal value of $\mathrm{SpO} 2(94.8 \%)$ and $\mathrm{PaO} 2 / \mathrm{FiO} 2(326 \mathrm{mmHg}$ ), but a high A-a gradient (37.1 $\mathrm{mmHg}$ instead of $15.5 \mathrm{mmHg}$ expected for age) suggesting an oxygen transfer / gas exchange problem, as usually detected in interstitial diseases, alveolar membrane diseases or V/Q mismatch.

Electrocardiogram documented regular sinus rhythm with normal ventricular repolarization. Echocardiography was normal with respiratory collapse of the inferior vena cava of more than 50\%. LUS showed diffuse B-lines in all the 12 fields examined (FIG. 2). Compression ultrasound of both the legs excluded DVT. Walking test documented a significant decrease in $\mathrm{SpO} 2$ value, from $95 \%$ at rest to $89 \%$ after 1 minute.



FIG. 2. Point-of-care lung US. The image shows B-pattern as expression of interstitial syndrome.

Blood tests showed normal blood counts, hepatic and liver function, serum electrolytes and glucose. C-reactive protein and LDH were slightly increased (respectively, $0.74 \mathrm{mg} / \mathrm{dL}$, normal value $0-0.5 ; 261 \mathrm{U} / \mathrm{L}$, normal value $0-248$ ). Troponin I was in the normal range $(3.9 \mathrm{ng} / \mathrm{L}$, normal value <31). D-dimer resulted highly increased $(5047 \mathrm{ng} / \mathrm{mL}$, normal value <500) with normal coagulation time and no significant change in fibrinogen value $(418 \mathrm{mg} / \mathrm{dL}$, normal value $150-400)$. We decided to perform an urgent nasopharyngeal swab, that resulted positive. Laboratory findings are reported in TABLE 1.

In order to investigate pulmonary embolism and interstitial damage due to a severe COVID-19 pneumonia, CT pulmonary angiography and high resolution chest CT were both performed and showed bilateral basal pulmonary microembolism (PE), ground-glass opacities and crazy-paving pattern (visual score 30\%-40\%) (FIG. 3 A and B). As consequence, the patient has been hospitalized in a COVID-19 ward with the diagnosis of COVID-19-related pulmonary embolism and persistent interstitial disease. 


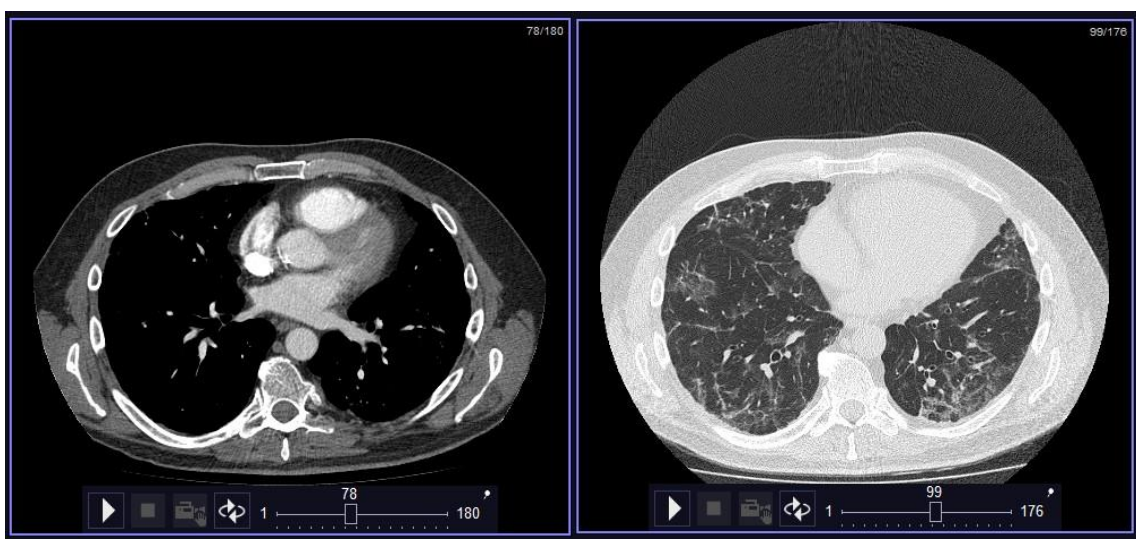

A

B

FIG. 3. Lung CT scan. The images show pulmonary embolism on CT pulmonary angiography (A, left), and multiple ground-glass opacities and and crazy-paving pattern (visual score $30 \%-40 \%)(\mathrm{B}$, right).

\section{Discussion}

COVID-19 can cause interstitial pneumonia with different grades of respiratory failure. Some patients can develop ARDS with the need of prompt NIV/CPAP or intubation. Special attention should be given to these patients after their release because no data about secondary consequences and persistent pro-thrombotic state are available in literature. At discharge LMWH should be considered for each patient. In addition, we recommend to always perform two consecutive nasopharyngeal swabs at 24-48 hours interval to confirm the negative result. As reported in literature, a single negative nasopharyngeal swab does not rule out COVID-19 [9,10], particularly in patients with persistent respiratory symptoms after adequate treatment of COVID-19, as reported in our case. In the next future, serology for COVID-19 should be useful for confirming the complete recovery from COVID-19 infection [11].

Finally, we strongly believe that all the patients discharged with the diagnosis of COVID-19 pneumonia should be monitored over time with a tailored and combined pneumological, cardiological and radiological follow-up in order to evaluate persistent organ damage, such as pulmonary fibrosis, and the development of pulmonary hypertension and right-sided heart failure (cor pulmonale), particularly in young patients even in absence of severe hypoxia.

\section{Acknowledgments}

The Authors are in debt with all the staff of the Emergency Department of the "Guglielmo da Saliceto" Hospital in Piacenza, for their high levels of involvement, commitment, responsibility, accountability, adaptability, flexibility, care, and dedication to work during COVID-19 epidemic.

\section{Conflicts of Interest}

The Authors declare that there are no competing interests 


\section{REFERENCES}

1. Poggiali E, Mateo RP, Bastoni D, et al. Abdominal Pain: A Real Challenge in Novel COVID-19 Infection. Eur J Case Rep Intern Med. 2020;7(4):001632.

2. Huang C, Wang Y, Li X, et al. Clinical features of patients infected with 2019 novel coronavirus 133 in Wuhan, China. Lancet. 2020;395(10223):497-506.

3. Zheng YY, Ma YT, Zhang JY, et al. COVID-19 and the cardiovascular system. Nat Rev Cardiol. 2020;17:259-60.

4. Wang $\mathrm{W}, \mathrm{Xu} \mathrm{Y}$, Gao R, et al. Detection of SARS-CoV-2 in different types of clinical specimens. Jama. 2020;323(18):1843-4.

5. Poggiali E, Dacrema A, Bastoni D, et al. Can lung ultrasound help critical care clinicians in the early diagnosis of novel coronavirus (COVID-19) pneumonia? Radiology. 2020; Mar 13:200847.

6. Pascarella G, Strumia A, Piliego C, et al. COVID-19 diagnosis and management: a comprehensive review. J Intern Med. 2020; Apr 29.

7. Poggiali E, Bastoni D, Ioannilli E, et al. Deep Vein Thrombosis and Pulmonary Embolism: Two Complications of COVID-19 Pneumonia? Eur J Case Rep Intern Med. 2020;7(5).

8. Bikdeli B, Madhavan MV, Jimenez D, et al. COVID-19 and Thrombotic or Thromboembolic Disease: Implications for Prevention, Antithrombotic Therapy, and Follow-up. J Am Coll Cardiol. 2020. pii:S0735-1097(20)35008-7.

9. Winichakoon P, Chaiwarith R, Liwsrisakun C, et al. Negative Nasopharyngeal and Oropharyngeal Swabs Do Not Rule Out COVID-19. J Clin Microbiol. 2020;58(5). pii:e00297-20.

10. Ai T, Yang Z, Hou H, et al. Correlation of Chest CT and RT-PCR Testing in Coronavirus Disease 2019 (COVID19) in China: A Report of 1014 Cases. Radiology. 2020; Feb 26:200642.

11. Tang YW, Schmitz JE, Persing DH, Stratton CW. The Laboratory Diagnosis of COVID-19 Infection: Current Issues and Challenges. J Clin Microbiol. 2020; Apr 3. pii: JCM.00512-20. 